\title{
Pitfalls in vascular imaging when brain death is suspected: multiparametric multidetector computed tomography as a complementary diagnostic tool
}

Armadilhas em imagem vascular na suspeita de morte encefálica: tomografia computadorizada multidetectores como uma ferramenta diagnóstica complementar Douglas Mendes Nunes ${ }^{1}$, Antônio José da Rocha ${ }^{1}$, Reginaldo Carlos Boni ${ }^{2}$

Although multidetector computed tomography (MDCT) has not yet been adopted to confirm brain death in Brazil, international reports support the use of multiparametric techniques to properly evaluate the circulation of the brain ${ }^{1}$.

Proposed MDCT protocol consists of two acquisitions to assess the arterial and venous phases of brain circulation. The complete absence of intracranial vascular enhancement supports the diagnosis of brain death. However, delayed, weak and persistent opacification of the proximal segments of the cerebral arteries, termed "stasis filling," indicates the inability to perform brain perfusion and should be recognized as an important pitfall when computed tomography angiography (CTA) is analyzed ${ }^{2,3}$.

Computed tomography perfusion (CTP) is reliable in demonstrating the absence of brain perfusion and thereby confirming severe hemodynamic arrest ${ }^{4,5}$ (Figure).
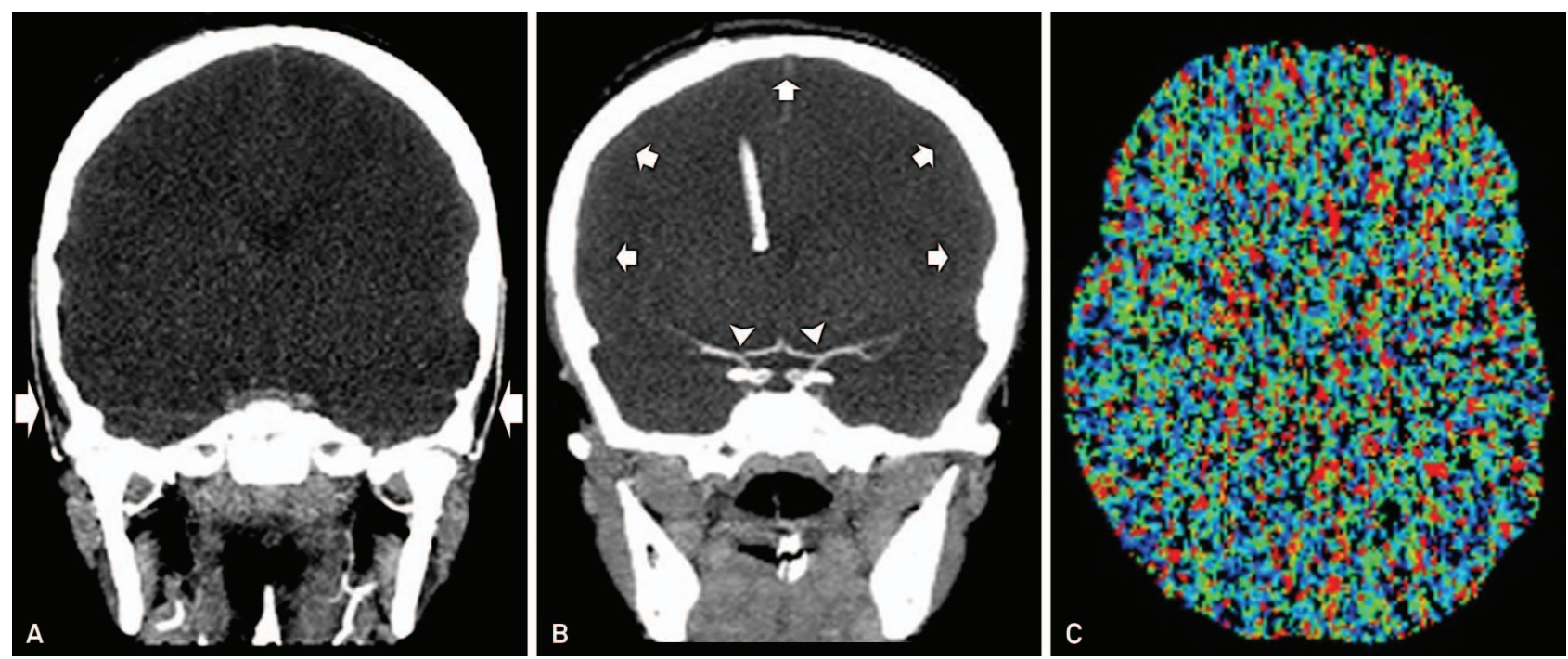

Figure. Multiparametric multidetector computed tomography of a 7-year-old boy with increased intracranial pressure following an intracranial hemorrhage (not shown) in the setting of acute lymphoblastic leukemia. A coronal reformatted image acquired in the first acquisition of CTA showed absent contrast opacification of the intracranial arteries (A). Note the extracranial arteries (arrows). The second acquisition showed "stasis filling" (arrowheads) of enhanced proximal cerebral arteries (B). Note the absence of contrast in the cortical vessels and venous drainage (arrows). The superior ophthalmic veins were ectatic (not shown). Microvascular evaluation by CTP revealed the absence of cerebral blood flow (C) and cerebral blood volume (not shown) in this patient, who was later confirmed to be brain dead according to Brazilian technical and legal determinations.

'Setor de Neurorradiologia, Santa Casa de Misericórdia de Sao Paulo, Sao Paulo SP, Brazil;

${ }^{2}$ Serviço de Procura de Órgãos e Tecidos, Santa Casa de Misericórdia de São Paulo, Sao Paulo SP, Brazil.

Correspondence: Antonio Jose da Rocha,Rua Dr. Cesário Motta Junior, 112; 01221-020 Vila Buarque, São Paulo SP, Brasil; E-mail: a.rocha@uol.com.br Conflict of interest: There is no conflict of interest to declare.

Received 23 January 2014; Received in final form 21 March 2014; Accepted 11 April 2014. 
1. Societe francaise de neuroradiologie, Societe francaise de radiologie, Agence de la biomedecine. Recommendations on diagnostic criteria of brain death by the technique of CT angiography. $J$ Neuroradiol 2011;38:36-39.

2. Dupas B, Gayet-Delacroix M, Villers D, Antonioli D, Veccherini MF, Soulillou JP. Diagnosis of brain death using two-phase spiral CT. AJNR Am J Neuroradiol 1998;19:641-647.
3

Sawicki M, Bohatyrewicz R, Safranow K, et al. Dynamic evaluation of stasis filling phenomenon with computed tomography in diagnosis of brain death. Neurorradiology 2013;55:1061-1069.

4. Shankar JJ, Vandorpe R. CT perfusion for confirmation of brain death. AJNR Am J Neuroradiol 2013;34:1175-1179.

5. Bohatyrewicz R, Sawicki M, Walecka A, et al. Computed tomografic angiography and perfusion in the diagnosis of brain death. Transpl Proc 2010;42:3941-3946. 